\title{
DYNAMIC CONFIGURATION OF THE COMPUTING NODES OF THE ALICE O2 SYSTEM
}

\author{
Summer Student Report | 7 August 2015 \\ Krittaphat Pugdeethosapol \\ Department of Computer Engineering \\ King Mongkut's University of Technology Thonburi, Thailand \\ Supervisors \\ Vasco Chibante Barroso \\ ALICE, CERN \\ Dr. Khajonpong Akkarajitsakul \\ Department of Computer Engineering \\ King Mongkut's University of Technology Thonburi, Thailand
}

\section{Overview}

ALICE (A large Ion Collider Experiment) [1] is a heavy-ion experiment located at CERN's LHC over the French-Swiss border. It is designed particularly to study the properties of Quark-Gluon Plasma (QGP) through using the collision of Proton-Proton, Nucleus-Nucleus, and ProtonNucleus at high energies.

In 2020, the ALICE experiment will restart collecting data with an upgraded detector, which is expected to handle $50 \mathrm{KHz}$ of $\mathrm{Pb}-\mathrm{Pb}$ interactions. A new computing system [2] is being designed (ALICE $\mathrm{O}^{2}$ ) to support these upgraded detectors. The data rate from the collisions is anticipated to be greater than $1 \mathrm{TBytes} / \mathrm{s}$ for $\mathrm{Pb}-\mathrm{Pb}$ events, roughly 100 times more than the current running rate. The purpose of the new system is to reduce the data volume read out from the detector in order to minimize the cost and requirements for data processing, and storage on the computing system. The concept of the $O^{2}$ system consists in implementing an online reconstruction and archival of all reconstructed data to permanent data storage. The data reduction will be achieved in-memory during data collection. The raw data will first be reconstructed by using online clustering and initial fast tracking. Then, it will be archived to data storage.

The $O^{2}$ computing system will be a high-throughput system consisting of many heterogeneous computing platforms similar to many high performance computing centers. One main component of the $\mathrm{O}^{2}$ system is the CCM (Control, Configuration and Monitoring) [3]. The CCM is responsible for supporting the shift crews and automatizing day-to-day operations. It will need to handle a large number of processes foreseen for the $O^{2}$ system (up to hundreds of thousands). The control system's job is to coordinate all processes in the system. The configuration system is used for setting both application and environment parameters. Lastly, the monitoring system is used for gathering information from the processes, identifying unusual patterns, and raising alarms. The overview of $O^{2}$ s CCM system is illustrated in Figure 1.1.1. 


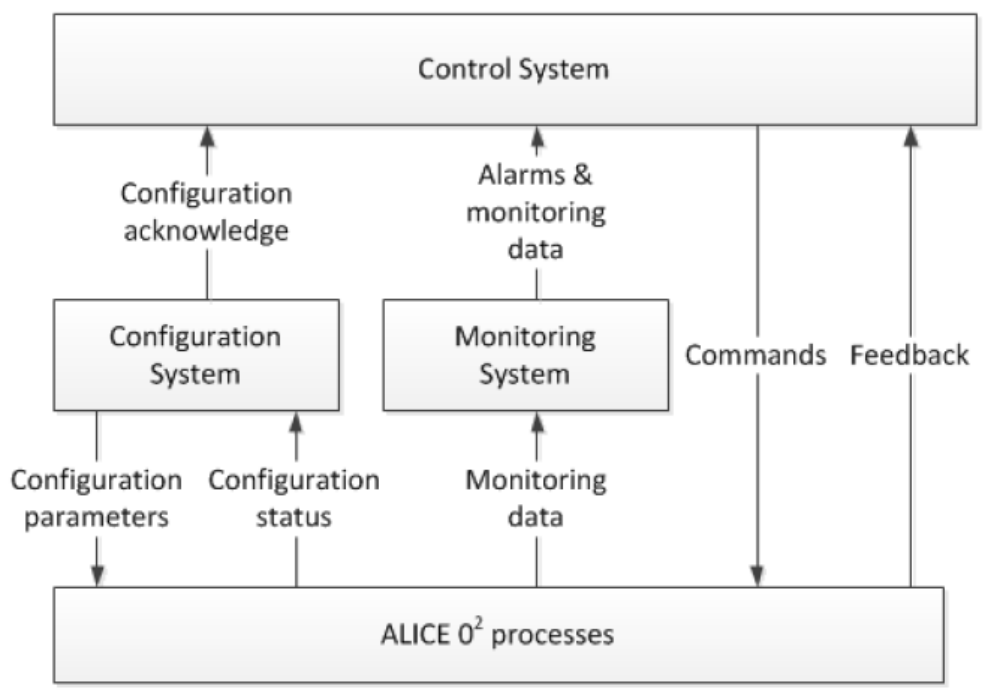

Figure 1.1.1 Overview of $O^{2}{ }^{\text {s }}$ CCM system [3]

\section{Scope of Work}

The topic of this research is the configuration system. Since $3^{\text {rd }}$ party tools will handle machine installation, network boot, automatic installation of base operating system, and package management, the research will mainly focus on $O^{2}$ process configurations which can be static and/or dynamic. In the static configuration, each process is restarted in order to read the new configuration. On the other hand, parameters in the dynamic configuration are loaded on-the-fly without stopping and restarting the process. In both modes, the Configuration system is responsible for distributing the configuration from the central repository to all processes in the system. The static configuration is supported by the operating system. However, for the dynamic configuration, we need to find the best solution to change parameters of each running process on the fly.

\section{Design of Configuration System}

\subsection{Overall System Design}

The overall system design is based on the model shown in Figure 3.1.1. The design includes 20 detectors, 250 First Level Processors (FLPs), 1500 Event Processing Nodes (EPNs), a frontend server, and a control room.

In order to read the collision data from the detectors, the FLPs machines must connect to the detectors via optical links. Afterward, the data from FLPs will be shipped to the EPN cluster for data reconstruction and archival. All the machines are connected to each other via an interconnection network. To control all the machines, the administrator or user will issue commands through the frontend server from the control room. From the architecture, we design the configuration system as a centralized server model. For the configuration system, we will focus on the frontend server, EPNs and FLPs machines. The configuration system will also have a configuration repository which is used to keep configuration files for the processes. This 
configuration repository machine will be connected with all the machines through the interconnection network. The frontend server is designed to manage the configuration parameters in the repository and also distribute the configuration parameters to the processes that are running on FLPs and EPNs. FLPs and EPNs will regularly update the configuration parameter with the server.

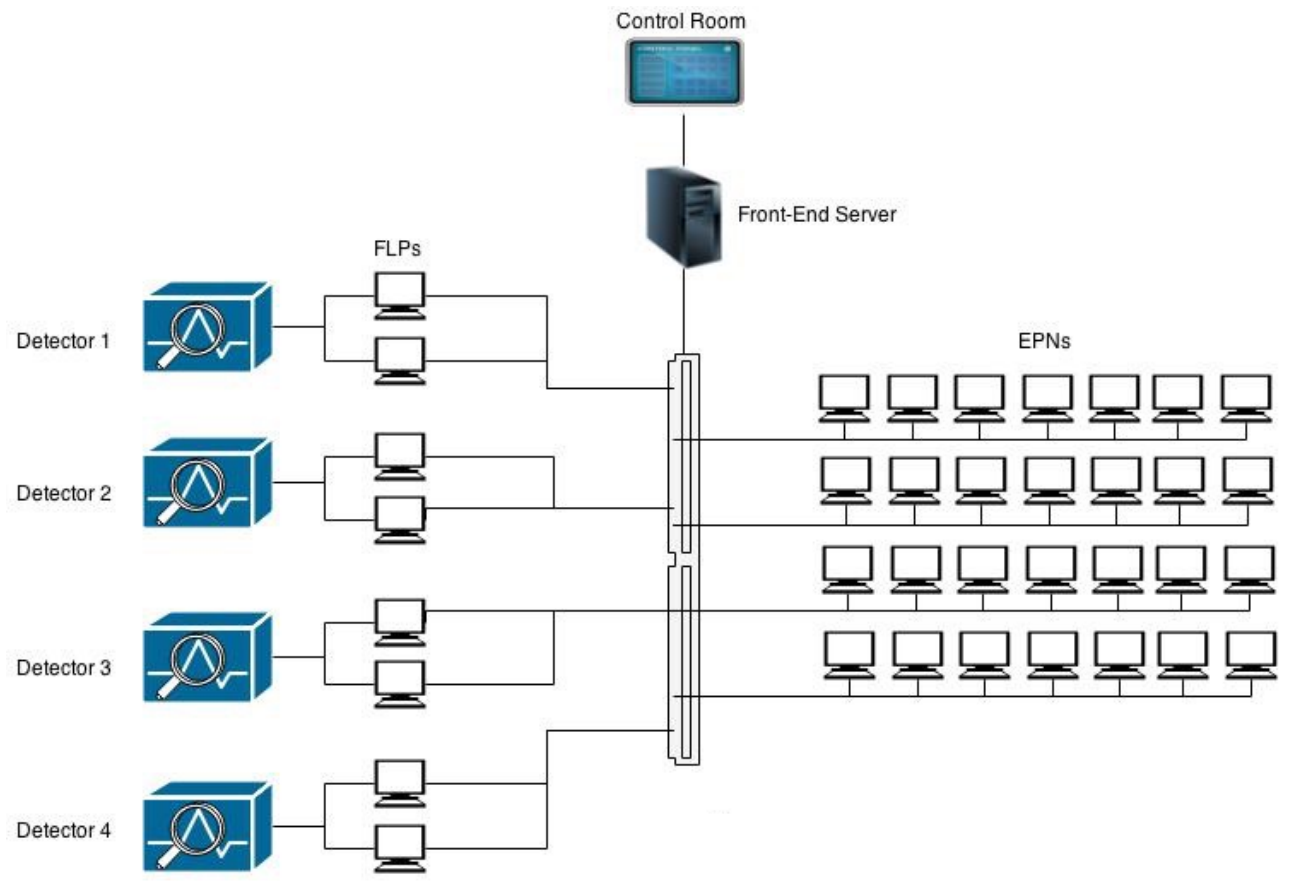

Figure 3.1.1 Overall system design

\subsection{Component Architecture}

Figure 3.2 shows the component architecture of the system which consists of server, agent, repository and external components. The server, agent, and repository components will be used to set application parameters. The external component will be used to set environment parameters for machine installation or network boot.

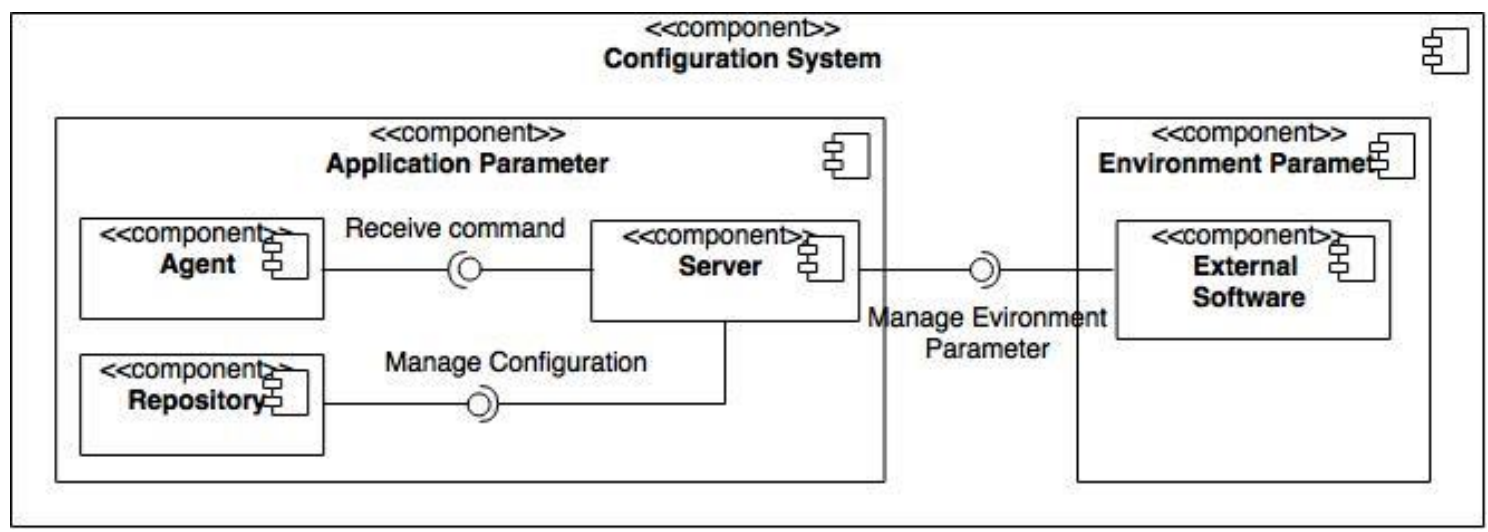


Figure 3.2 Component Architecture

\subsection{Frontend Server}

The frontend server is the main component of the system. It is used to interact with users or administrators. This frontend server will provide a web based interface for users to manage the configuration. The web interface will be developed using HTML5, Javascript, and CSS. Via the web based GUI, users will be able to:

- define, save, load, or propagate configurations

- validate input parameters

The web based interface will also show:

- status of deployed configuration (failed, pending or finished)

- history of deployed configuration

- actual configuration parameters.

The server will receive a command from a user and communicate with the configuration repository in order to manage a configuration file. Additionally, the server will communicate with each client to update its configuration and also get the status of updated configurations.

\subsection{Client (EPNs/FLPs)}

The client machines will run processes of the $O^{2}$ system. The total number of processes that will run on the system is estimated to be up to $70 \mathrm{~K}$. This means that each machine will contain many processes running on it. On each machine there will be a broker that can communicate with the server to receive messages or commands and communicate with the configuration repository to receive the configuration file. The broker will also be able to control each process in that machine. The broker can start, stop, restart, or update the configuration parameters. The architecture of client machine is show in the Figure 3.4.1.

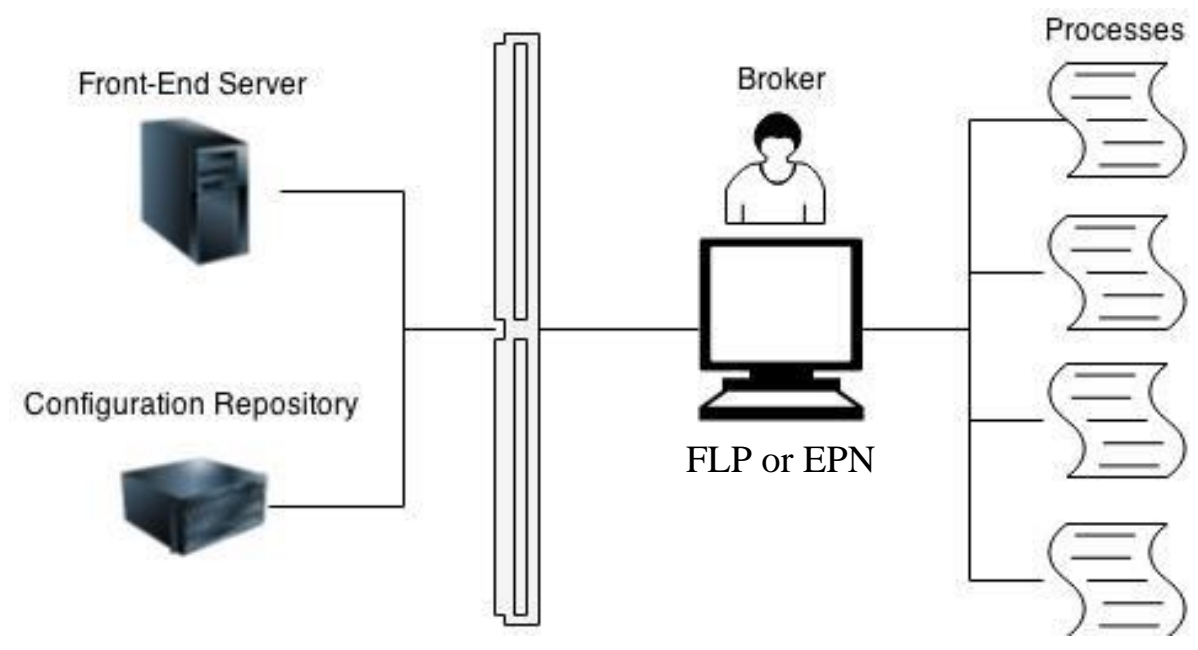

Figure 3.4.1 Architecture of client machine 
A block of code needs to be inserted on each process that runs on the machine in order to receive a command from the broker. The flow of code is shown in Figure 3.4.1. The process will check and read a new parameter file only when it receives a signal from the broker.

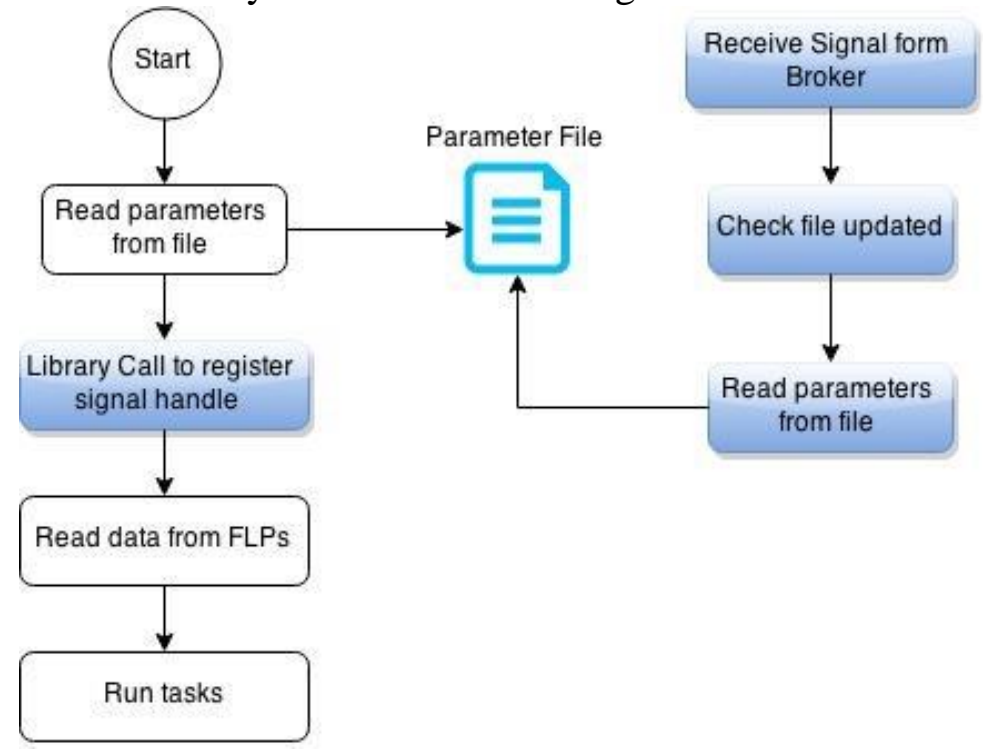

Figure 3.4.2 Flow of code

\subsection{Configuration Parameters and Distribution}

Configuration parameters of each process will be kept in a key/value-based file in the configuration repository. Each configuration node will be named by the process using the configuration. The configuration repository will manage all the configuration of the process that is running on the system.

Configuration parameters in the configuration file kept in the configuration repository will be read by the broker after the server receives the command from the user. The frontend server will send a command to the broker by using the widely used ZeroMQ interprocess communication library. The broker of each machine will receive the command to read the parameter from the repository and store it in its local directory. Then the broker will send a signal to the process so that the process will read new configuration parameters.

\section{Experimental and Result}

I have started working with the configuration repository tools. The tools must be able to distribute the configuration from a central repository to all processes in the system and handle a large volume of configuration data. I have reviewed 3 tools: etcd, consul, and zookeeper.

\subsection{Etcd}

Etcd is one of the components of the open source project for Linux container called CoreOS [4]. Etcd is a distributed key/value storage that provides shared configuration and service discovery. It can run on a single machine or as a cluster. An application can read and write data into etcd by using a simple HTTP library like curl. Values are stored in an hierarchical structure similar to a 
file system. It also has a watch feature which allows to watch for key changes and react to new values. Etcd claims that it can handle 1000s of write operations per second per instance.

\subsection{Consul}

Consul is a tool for discovering and configuring services in infrastructure [5]. It has many features like service discovery, health checking and multi datacenter. Key value storage is one of Consul's features in which we are interested. The application can make use of Consul's key value storage to do many things like dynamic configuration, feature flagging, coordination, leader election, and others. The main interface for using Consul is a RESTFul HTTP API. It also has a web interface for human interaction.

\subsection{Zookeeper}

Zookeeper [6] is an open-source software that can be used for maintaining configuration information, naming, providing distributed synchronization, and providing group services. The data model and the hierarchical namespace of the Zookeeper are what we are interested in. The namespace is similar to that of a standard file system. However, Zookeeper supports only Java or $\mathrm{C}$ binding, so it imposes a programming language limitation.

I went through the tutorial of these 3 tools to gain some experience and compare them. Etcd and consul use a HTTP-based API which is easy to use compared to zookeeper that uses either a Java-based or script API. Since etcd is designed specifically to serve as a key/value store and additionally includes a watch function, I've selected etcd.

I have set up 1 single server and 23 clients. All 23 clients have scripts to run the processes that read the parameter value of the same key from the server. I started testing with 100 processes and went up to 10,000 processes per machine to see the different. The experiment aims to measure the average time that each process uses to read the value. The results are shown in the table and graph below.

\begin{tabular}{|cccc}
$\begin{array}{c}\text { Number of } \\
\text { Process per } \\
\text { Machine }\end{array}$ & Time (sec) & $\begin{array}{c}\text { Number of } \\
\text { Process per } \\
\text { Machine }\end{array}$ & Time (sec) \\
\hline 100 & 0.0048 & 4500 & 9.10 \\
\hline 200 & 0.0055 & 5000 & 13.48 \\
\hline 300 & 0.0061 & 5500 & 12.19 \\
\hline 400 & 0.0066 & 6000 & 14.72 \\
\hline 500 & 0.0078 & 6500 & 17.40 \\
\hline 1000 & 0.60 & 7000 & 20.30 \\
\hline 1500 & 1.05 & 7500 & 21.10 \\
\hline 2000 & 2.90 & 8000 & 23.42 \\
\hline 2500 & 3.19 & 8500 & 25.77 \\
\hline 3000 & 4.38 & 9000 & 27.33 \\
3500 & 4.98 & 9500 & 33.04 \\
\hline 4000 & 7.69 & 10000 & 44.82 \\
\hline
\end{tabular}


Table 4.1 etcd average read time

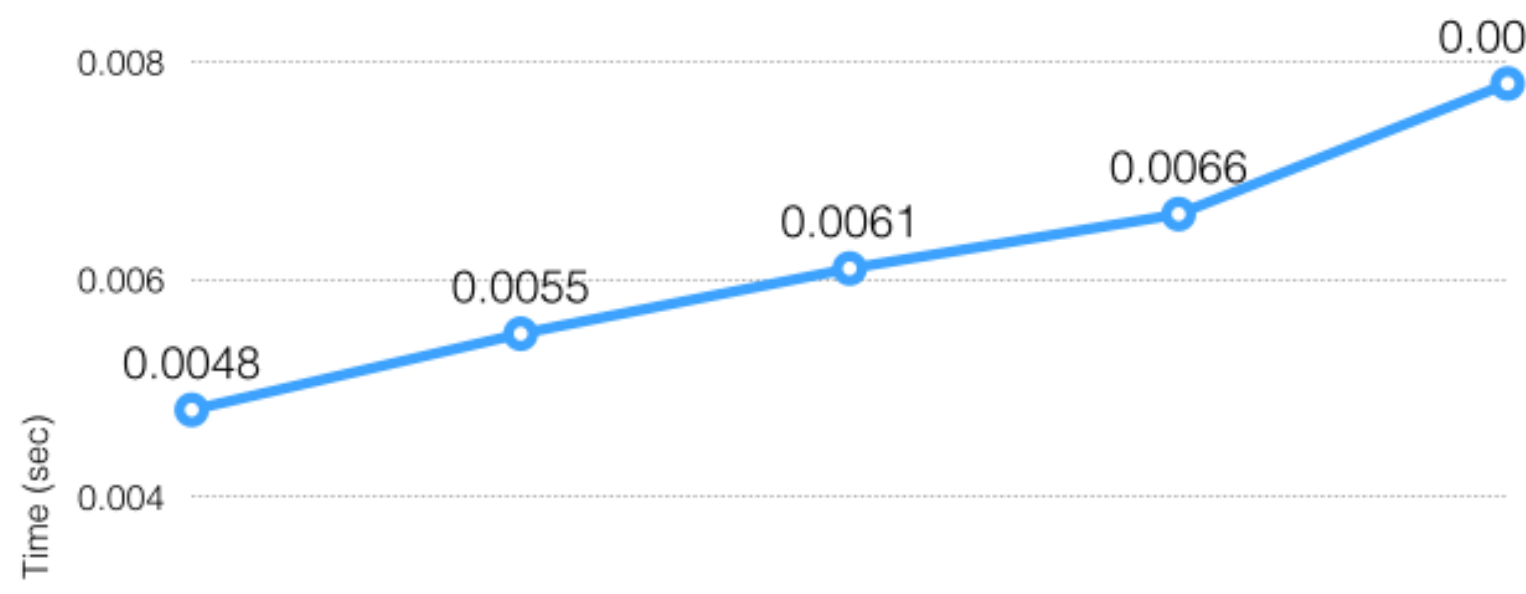

0.002

$\begin{array}{cccc}0 & 300 & 400 & 500 \\ & & & \\ & \text { Parameters } & & \end{array}$

50

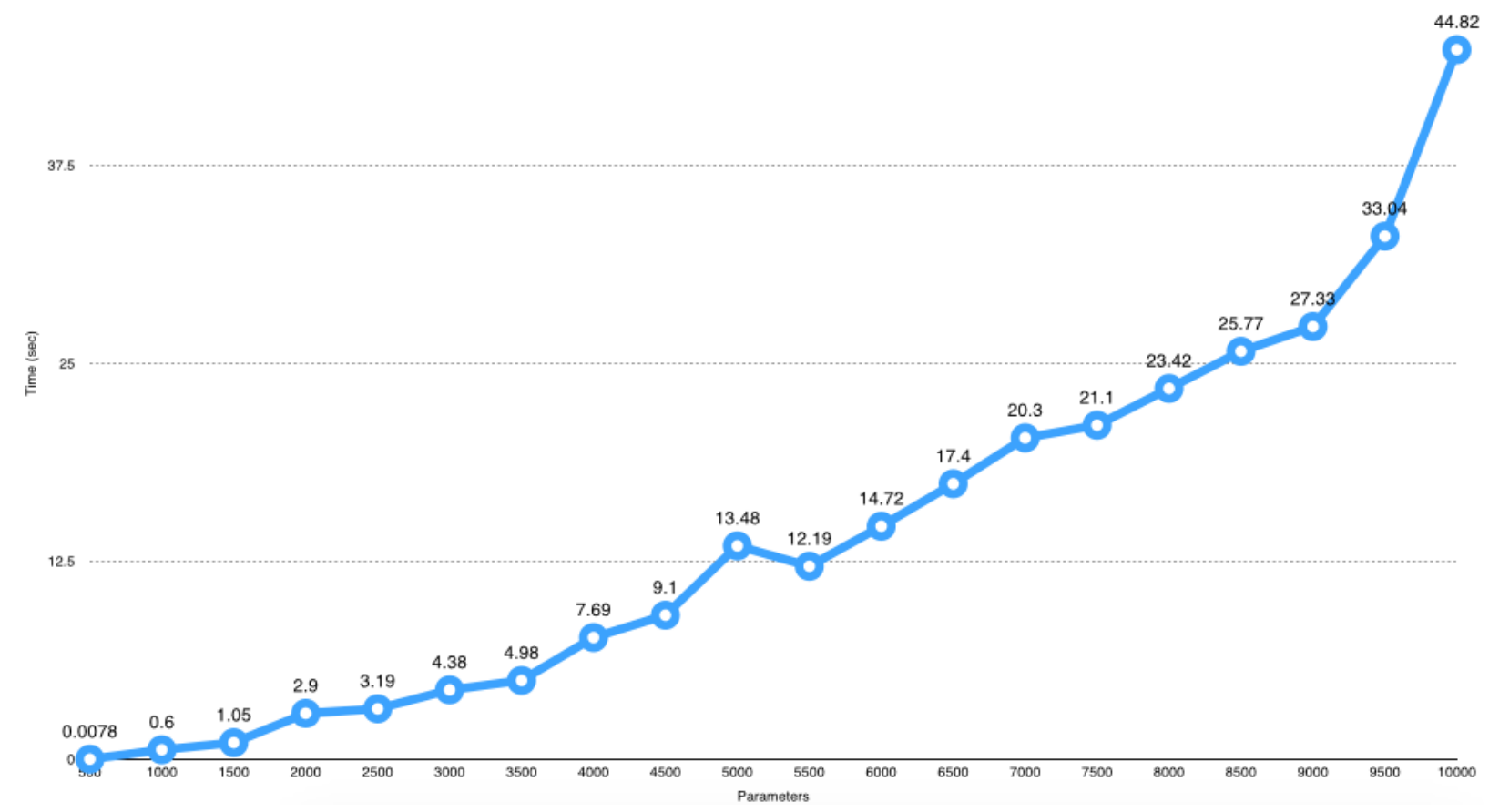

Figure 4.2 Average time each process use to read parameter value 


\section{Conclusion}

Collaborating with ALICE, we will design the configuration system which is a part of the overall Control, and Configure and Monitoring (CCM) system. We have proposed the designed system that is used to distribute the configuration from a central repository to processes in the $O^{2}$ system. The system can be used with both static and dynamic configuration modes. After designing the system, we will implement a prototype in order to get the performance analysis. The experiment will mainly focus on the time used by each process to update its configuration and the overall time to configure the whole system. We hope that the proposed framework will fulfill the aim of this thesis which is to design a configuration system and find the best solution for distributing configuration parameters and changing parameters of each running process on the fly.

\section{References}

[1] ALICE Collaboration, 2008, "The ALICE experiment at the CERN LHC", 2008 JINST 3 S08002.

[2] ALICE Collaboration, 2015, "Technical Design Report for the Upgrade of the OnlineOffline System", CERN-LHCC-2015-006/ALICE-TDR-019.

Available : http://cds.cern.ch/record/2011297

[3] ALICE Collaboration, 2014, "ALICE CWG10-Control, Configuration and Monitoring Software Requirements Specifications".

[4] Etcd

Available : https://coreos.com/etcd

[5] Consul

Available : https://consul.io

[6] Zookeeper

Available : https://zookeeper.apache.org 